\title{
Next-to-leading order corrections to light-quark mixed QCD-EW contributions to Higgs boson production
}

\author{
Matteo Becchetti॰* \\ Dipartimento di Fisica, Università di Torino and INFN Sezione di Torino, \\ Via Pietro Giuria 1, I-10125 Torino, Italy \\ Roberto Bonciani ${ }^{\dagger}$ \\ Dipartimento di Fisica, Università di Roma "La Sapienza" and INFN Sezione di Roma, \\ Piazzale Aldo Moro 2, 00185 Roma, Italy \\ Vittorio Del Duca $\oplus^{\ddagger}$ \\ ETH Zürich, Institut für theoretische Physik, Wolfgang-Pauli Straße 27, 8093, Zürich, Switzerland \\ and INFN, Laboratori Nazionali di Frascati, 00044 Frascati (RM), Italy \\ Valentin Hirschi $\odot,{ }^{\S}$ Francesco Moriello, ${ }^{\|}$and Armin Schweitzer ${ }^{\llbracket}$ \\ ETH Zürich, Institut für theoretische Physik, Wolfgang-Pauli Straße 27, 8093 Zürich, Switzerland
}

(Received 30 October 2020; accepted 23 February 2021; published 26 March 2021)

\begin{abstract}
We present for the first time the exact next-to-leading-order QCD corrections to the light-quark part of the mixed QCD-EW contributions to Higgs production via gluon fusion at the Large Hadron Collider, for a center-of-mass energy of $13 \mathrm{TeV}$, with exact EW-boson mass dependence. The relevant two-loop realemission matrix element is computed using a dynamic one-dimensional series expansion strategy whose stability and speed allows for a numerical phase-space integration using local IR subtraction counterterms. For the choice of renormalization $\left(\mu_{R}\right)$ and factorization $\left(\mu_{F}\right)$ scales, $\mu_{R}=\mu_{F}=M_{H}$, we find $\sigma_{g g \rightarrow H+X}^{\left(\alpha_{s}^{2} \alpha^{2}+\alpha_{s}^{3} \alpha^{2}\right)}=1.467(2)_{-14.6 \%}^{+18.7 \%}\left(\mu_{R} \operatorname{var}\right) \pm 2 \%(\mathrm{PDF}) \mathrm{pb}$, which we use to provide the best result, including an estimate of suppressed contributions: $\sigma_{p p \rightarrow H+X}^{(\text {EW,best) }}=2.11 \pm 0.28$ (theory) pb.
\end{abstract}

DOI: $10.1103 /$ PhysRevD.103.054037

\section{INTRODUCTION}

After the discovery of the Higgs boson with the Large Hadron Collider (LHC) at CERN in 2012 [1,2], the work of the LHC community has focused on the study of the Higgs sector, which provides a stringent test of the Standard Model of particles (SM) and a fertile environment for the search of new physics (NP) signals [3-15].

A key ingredient for predictions of Higgs observables is accurate knowledge of the Higgs production cross section in gluon fusion, which at the LHC is by far the dominant

\footnotetext{
*matteo.becchetti@unito.it

roberto.bonciani@roma1.infn.it

*delducav@itp.phys.ethz.ch

§valentin.hirschi@gmail.com

"fmoriell@itp.phys.ethz.ch

ๆarmin.schweitzer@phys.ethz.ch
}

Published by the American Physical Society under the terms of the Creative Commons Attribution 4.0 International license. Further distribution of this work must maintain attribution to the author(s) and the published article's title, journal citation, and DOI. Funded by SCOAP . production mode. The coupling of the Higgs boson to gluons is mediated by a heavy-quark loop. The Higgs production cross section was computed at leading order in the 1970s [16], and at next-to-leading order (NLO) in the strong coupling constant $\alpha_{s}$ in the 1990s [17,18]. NLO QCD corrections are sizable ( $80 \%-100 \%)$, undermining the reliability of the perturbative expansion in $\alpha_{s}$ of the production cross section. The next-to-next-to-leading-order (NNLO) [19-21] and the next-to-next-to-next-to-leadingorder $\left(\mathrm{N}^{3} \mathrm{LO}\right)[22,23]$ corrections in $\alpha_{s}$ have been computed in the Higgs effective field theory (HEFT) approach-i.e., in the limit of a top quark much heavier than the Higgs boson, $M_{T} \gg M_{H}$, with all other quarks taken as massless, which replaces the loop-mediated coupling with an effective tree-level coupling. The NNLO corrections turn out to be significant $(\sim 10 \%-20 \%)$, but with a reduced scaledependent uncertainty. The $\mathrm{N}^{3} \mathrm{LO}$ corrections turn out to be small $(\sim 4 \%-6 \%)$ [24], with a renormalization/factorization scale variation of less than $2 \%$.

The high accuracy of the $\mathrm{N}^{3} \mathrm{LO}$ corrections calls for the evaluation of finite quark-mass effects and electroweak 
contributions. Finite quark-mass effects are known through NLO [18,25-30] and contribute a $\sim-7 \%$ change [29] to the cross section. Although the relevant ingredientsdouble-virtual [31-33], real-virtual [34-36], and doublereal $[37,38]$ — of the computation of the finite quark-mass effects at NNLO are available, such a computation has not been performed yet. At NNLO, top-quark mass effects have been estimated through a power expansion in $M_{H} / M_{T}$ [39-41] and found to be $\sim 1 \%$. Light-quark mass effectsin particular, the top-bottom interference-are not yet known at NNLO.

Mixed QCD-electroweak (EW) effects arise at two loops ${ }^{1}$-i.e., at $\mathcal{O}\left(\alpha^{2} \alpha_{s}^{2}\right)$. They are due to the gluons coupling to EW bosons $V=W, Z$ through a quark loop, followed by the gauge coupling of the EW bosons to the Higgs boson. Mixed QCD-EW contributions were calculated for the light-quark loop [42-44], for the heavy-quark loop [45], and with full quark-mass dependence [45], and were found to increase the $\mathrm{N}^{3} \mathrm{LO}$ cross section by about $2 \%$ [24]. Since this increase is of the order of the residual QCD uncertainty, it is important to compute the NLO corrections in $\alpha_{s}$. Because the largest part ( 98\% [44]) of the increase at $\mathcal{O}\left(\alpha^{2} \alpha_{s}^{2}\right)$ is due to the light-quark part of the mixed QCDEW contributions, the evaluation of the NLO corrections has been aimed at the light-quark part. These corrections were evaluated in the limit where the Higgs mass is much smaller than the EW boson masses, $M_{H} \ll M_{V}$ [46], and they turned out to be sizable.

The $\mathcal{O}\left(\alpha^{2} \alpha_{s}^{3}\right)$ corrections consist of three parts: the oneloop $2 \rightarrow 3$, the three-loop $2 \rightarrow 1$, and the two-loop $2 \rightarrow 2$, with sample diagrams shown in the first column of Fig. 1. In Ref. [47], the one-loop $2 \rightarrow 3$ processes were computed and found to yield a negligible contribution. The three-loop contribution was evaluated analytically and expressed in terms of multiple polylogarithms (MPLs) [48]. In Ref. [49], the soft part of the two-loop $2 \rightarrow 2$ process was added. In Ref. [50], the total cross section was evaluated in the small EW-boson mass limit, $M_{V} \ll M_{H}$. The planar master integrals (MIs) for the two-loop $g g \rightarrow H g$ process with the exact EW-boson mass were published in Ref. [51] and recently, in Ref. [52], the complete helicity amplitudes, including the nonplanar diagrams, were presented. The calculation was done analytically, expressing the results in terms of MPLs.

In this work, we perform an independent computation of the amplitude and of the MIs, which are evaluated by using the series solution method of Ref. [53], and we present the NLO QCD corrections to the total cross section for Higgs production via gluon fusion at the LHC, due to the lightquark part of the mixed QCD-EW contributions, with exact EW boson mass dependence.

\footnotetext{
${ }^{1}$ We count all factorized coupling constants except the strong coupling as $\alpha$.
}

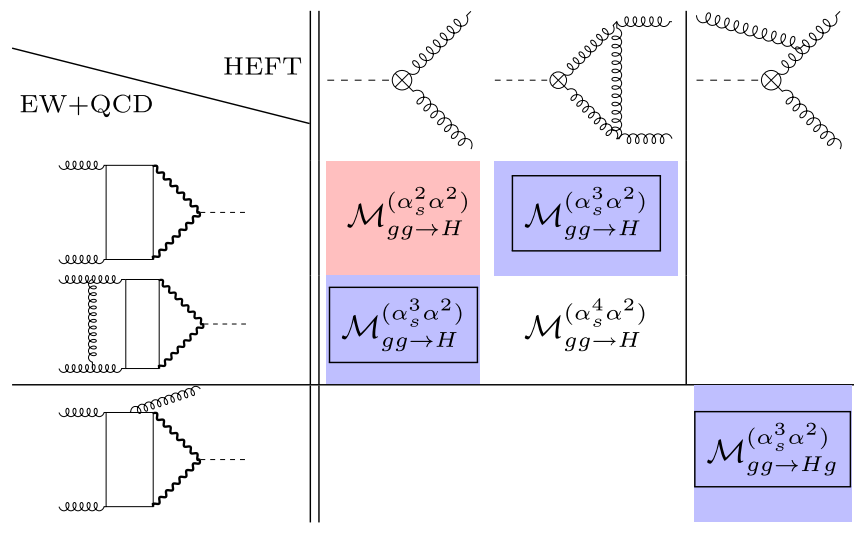

FIG. 1. Overview of the relevant interferences necessary for the computation of the hadronic cross section $\sigma_{g g \rightarrow H+X}^{\left(\alpha_{s}^{2} \alpha^{2}+\alpha_{s}^{3} \alpha^{2}\right)}$. The red cell is the LO, and cells highlighted in blue are part of the NLO contribution. Amplitudes are denoted by a single representative diagram. Curly lines denote gluons, wavy lines are massive weak gauge bosons, continuous straight lines are massless quarks, and the dashed line represents the Higgs boson.

\section{CALCULATION}

\section{A. Loop amplitudes}

The computation of the two-loop $g g \rightarrow H g$ amplitude for the matrix element $\mathcal{M}_{g g \rightarrow H g}^{\left(\alpha_{s}^{3} \alpha^{2}\right)}$ (see Fig. 1) is performed by using the series expansion method of Ref. [53]. Specifically, we reduce the amplitude to MIs by using computer programs [54-56] for the solution of integrationby-parts identities (IBPs) [57-59] and Lorentz-invariance identities (LI) [60]. By taking advantage of these identities, we define a system of differential equations [61-64] for a basis of canonical MIs [65]. The canonical basis is found by using the methods of Refs. [65-71], and the corresponding differential equations are solved in terms of generalized power series, as described in Ref. [53]. The system of differential equations uniquely defines the solution when imposing boundary conditions at a special kinematic point. We consider the infinite EW boson mass limit as our initial boundary point. The numerical evaluation of the relevant scattering amplitudes by means of the generalized power series approach is well suited for Monte Carlo phase-space integrations. Moreover, the analytic continuation of the generalized power series to the physical regions is fully algorithmic.

The series solution strategy can be summarized as follows: We transport the integrals from a known boundary point to a phase-space point of interest by solving the differential equations in terms of generalized power series along the line connecting the pair of points. This is done dynamically for every new phase-space point. When the line crosses a physical threshold, the analytic continuation is defined by assigning a vanishing imaginary part to the line parameter, in accordance with Feynman prescription. By construction, the nonanalytic terms of the series are 
logarithms and rational powers of the line parameter, and their analytic continuation is elementary. In order to improve the efficiency of the evaluations, we consider a precomputed grid of about 5000 physical phase-space boundary points. In this way, the series solution can be found along lines connecting pairs of points separated by a relatively short distance. In general, these lines cross fewer singular points of the differential equations, and they require, for fixed precision, a lower truncation order of the series, considerably reducing the average evaluation time. In this work, we consider truncated power series which guarantee a precision for the numerical evaluations of the integral basis of at least 16 digits after the decimal point. The average time for one form-factor evaluation on one CPU core is $\mathcal{O}(1 \mathrm{~min})$, ranging from $\mathcal{O}(30 \mathrm{sec})$ up to $\mathcal{O}(10 \mathrm{~min})$ for input kinematic configurations featuring large-scale hierarchies.

We verify our computation against Ref. [52] and report benchmark values for the relevant matrix elements in the Appendix. As for the three- and two-loop matrix elements of the $g g \rightarrow H$ process $\left[\mathcal{M}_{g g \rightarrow H}^{\left(\alpha_{s}^{3} \alpha^{2}\right)}\right.$ and $\mathcal{M}_{g g \rightarrow H}^{\left(\alpha_{s}^{2} \alpha^{2}\right)}$, respectively, in Fig. 1], we use the results presented in Refs. [48,72].

\section{B. Phase-space integration and infrared regularization}

Infrared (IR) divergences are locally subtracted using two different paradigms for cross-validation: first, the Frixione, Kunszt, and Signer (FKS) subtraction scheme [73] and second, a modified version [74,75] of the COLORFUL $[76,77]$ scheme at NLO. To the best of our knowledge, this is the first time that a two-loop matrix element with implicit phase-space IR divergences has been numerically integrated using local subtraction counterterms. To achieve this, we encoded the loop amplitudes discussed in the previous paragraph as form factors of effective vertices in the Universal FeynRules Output (UFO) [78] model. We then created a custom plugin made publicly available $^{2}$ for the MadGraph5_aMC@NLO program [79], allowing for the generation of a standalone library for the evaluation of all matrix elements entering our computation. We finally customized the generation output of MadGraph5_aMC@NLO for the NLO QCD correction of inclusive Higgs hadroproduction within the Higgs Effective Theory (HEFT) in order to accommodate the aforementioned matrix elements as well as an offline parallelization pipeline.

We validated our results against those obtained using a modified version of the NLO implementation [77] of the COLORFUL subtraction scheme rendered suitable for the computation of a Higgs-inclusive cross section and implemented in the private extension of MadGraph5_aMC@NLO already featured in Ref. [47]. In that variant, the local soft

\footnotetext{
${ }^{2}$ https://bitbucket.org/aschweitzer/mg5_higgs_ew_plugin/ or http://madgraph.physics.illinois.edu/Downloads/PLUGIN/ higgsew.tar.gz.
}

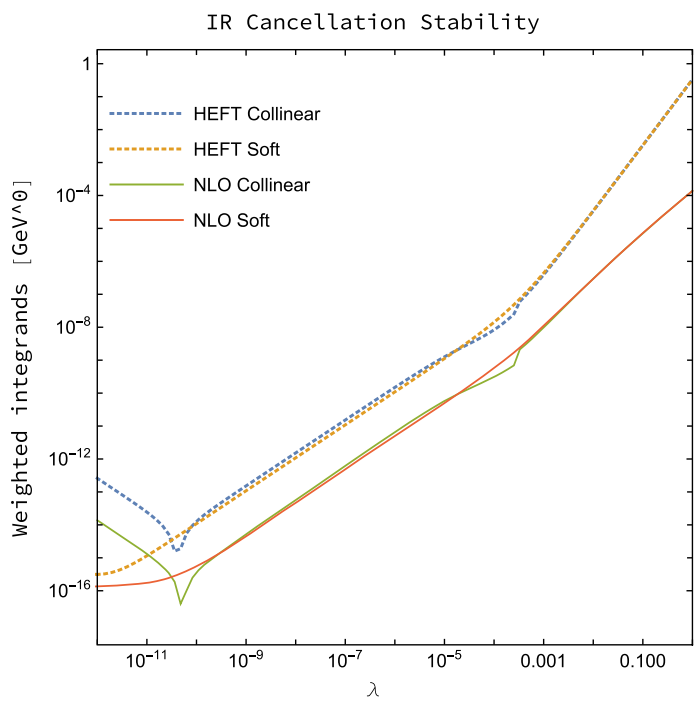

FIG. 2. Numerical stability of the two-loop real-emission matrix element, locally subtracted with our modified implementation of COLORFUL, compared to their HEFT tree-level counterpart when approaching the soft and collinear limits. The approach parameter $\lambda$ is defined so that the scaling of the real-emission matrix when approaching the IR limit is $\lambda^{-1}$. The weighted integrand shown includes the Jacobian of the parametrization so that it must scale like $\lambda^{\alpha}$ with $\alpha>\frac{1}{2}$ in order to be integrable.

counterterm uses a mapping recoiling against initial states (see Ref. [75] and Sec. 5.3.3 of Ref. [74]) and locally identically cancels against its soft-collinear counterpart. This is a consequence of the fact that our real-emission matrix element only features soft emission from an initialinitial dipole. The initial-final collinear counterterms alone are thus sufficient to regularize the infrared (IR) divergences involved in our computation. We showcase the stability of the real-emission matrix element in Fig. 2 by investigating the quality of its cancellation against local IR counterterms both in the collinear and soft limits. We find stability on par with that obtained in HEFT when considering tree-level real-emission matrix elements, where the limiting factor is the double-precision accuracy of the input kinematics. We also tested the independence of our result on the arbitrary cutoff of the local IR collinear counterterms (parameter $y_{0}^{\prime}$ in Ref. [77]). We verified that our two independent implementations of the FKS and COLORFUL IR subtraction procedure give consistent results.

\section{RESULTS}

We carry out our computation in the Standard Model (SM) using the relevant input parameters given in Table I, where the masses of the gauge bosons are chosen in order to align with the literature [48,72], and the weak coupling follows from the gauge relation with $G_{F}$. The Weinberg mixing angle is determined by $\cos \theta_{W}=M_{W} / M_{Z}$. 
TABLE I. Standard Model parameters used for obtaining all numerical results presented in this work. Dimensionful parameters are given in $\mathrm{GeV}$ unless indicated otherwise. All particle widths are set to zero.

\begin{tabular}{llll}
\hline \hline Parameter & \multicolumn{1}{c}{ Value } & Parameter & \multicolumn{1}{c}{ Value } \\
\hline PDF set & PDF4LHC15_nlo_30 & $\mu_{R}=\mu_{F}$ & $M_{H}{ }_{\pi \alpha}$ \\
$\alpha_{S}\left(\mu_{R}\right)$ & As per PDF set. & $G_{F}$ & $\frac{\sqrt{2} M_{W}^{2}\left(1-M_{W}^{2} / M_{Z}^{2}\right)}{}$ \\
$\sqrt{\hat{s}}$ & $13 \mathrm{TeV}$ & $\alpha^{-1}$ & 132.507 \\
$M_{Z}$ & 91.1876 & $V_{i j}^{\mathrm{CKM}}$ & $\delta_{i j}$ \\
$M_{W}$ & 80.3845 & $M_{H}$ & 125.09 \\
\hline \hline
\end{tabular}

We remind the reader that we consider for the $W$ exchange only the first two light-quark generations, whereas the $Z$-exchange also receives contributions from massless $b$ quarks, as discussed in Refs. [48,72]. We furthermore neglect the contribution from $g g \rightarrow H q \bar{q}$ (computed to be below $0.1 \mathrm{pb}$ in Ref. [47]).

For comparison purposes, we start by providing here the cross section for the hadroproduction of a Higgs boson, computed at LO and NLO QCD in HEFT:

$$
\begin{gathered}
\sigma_{g g \rightarrow H+X}^{\left(\mathrm{HEFT}, \alpha_{s}^{2} \alpha\right)}=13.209_{-17.3 \%-2.0 \%}^{+23.4 \%+2.0 \%} \mathrm{pb}, \\
\sigma_{g g \rightarrow H+X}^{\left(\mathrm{HEFT}, \alpha_{s}^{2} \alpha+\alpha_{s}^{3} \alpha\right)}=30.484_{-15.3 \%-1.9 \%}^{+19.8 \%+1.9 \%} \mathrm{pb},
\end{gathered}
$$

where the first set of uncertainties corresponds to the $\left(\frac{1}{2}, 1,2\right) \mu_{R}$ scale variation. The second set reports the PDF uncertainty obtained within MadGraph5_aMC@NLO, which uses the LHAPDF [80] interface. At this point, the factorization scale variation is insofar not a reliable error estimate, since we only consider initial-state gluons, and its size will therefore depend strongly on the chosen central scale. However, the reduction of the scale dependence can be seen in Eq. (20), which also includes an estimate for the $q g$ channel. Our result for the correction from light quarks to the mixed QCD-EW contribution to the inclusive Higgs production cross section is

$$
\begin{gathered}
\sigma_{g g \rightarrow H+X}^{\left(\alpha_{s}^{2} \alpha^{2}\right)}=0.68739_{-17.3 \%-2.0 \%}^{+23.4 \%+2.0 \%} \mathrm{pb}, \\
\sigma_{g g \rightarrow H+X}^{\left(\alpha_{s}^{2} \alpha^{2}+\alpha_{s}^{3} \alpha^{2}\right)}=1.467(2)_{-14.6 \%-2.0 \%}^{+18.7 \%+2.0 \%} \mathrm{pb} .
\end{gathered}
$$

The resulting pure NLO QCD correction of order $\mathcal{O}\left(\alpha_{s}^{3} \alpha^{2}\right)$ is $0.780(2) \mathrm{pb}$ and was obtained from 50000 evaluations of the real-emission matrix element in our private implementation of the COLORFUL NLO subtraction scheme. The gluon-initiated cross section with exact EW-boson mass dependence in the virtual contributions and the real contribution treated in the soft-gluon, the massless, and the infinite mass approximations increase the pure gluoninduced HEFT NLO cross section by 5.4\% [49], 5.4\% [50], and 5.2\% [50], respectively, whereas our exact

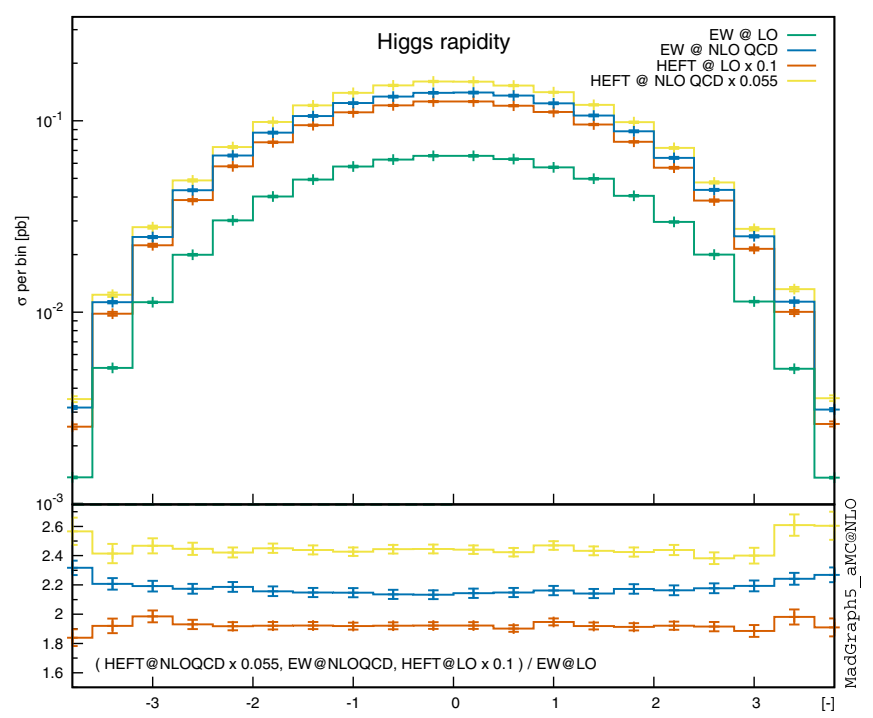

FIG. 3. Differential prediction for the $\mathcal{O}\left(\alpha_{s}^{3} \alpha^{2}\right)$ correction to the Higgs rapidity distribution.

computation, with $\mu_{R}=\mu_{F}=\frac{1}{2} M_{H}$ and $\alpha^{-1}=128.0$ as in Refs. $[49,50]$ yields $5.1 \%$. Our result therefore lies within the original uncertainty assigned to the factorization estimate of $5 \% \pm 1 \%$ given in Ref. [20] and used in Ref. [24]. We furthermore notice the small reduction of the scale uncertainty when including NLO corrections. This slow convergence is a known feature of gluon-fusion Higgs production.

We present the two most relevant differential predictions in Figs. 3 and 4. First, in Fig. 3, we show the NLO-accurate Higgs rapidity distribution, which reveals the expected flat differential $K$ factor, both for the EW effects with respect to the HEFT approximation and for the QCD corrections. Second, in Fig. 4, we show the Higgs transverse momentum distribution, which is accurate at leading order, and which we compare to its HEFT counterpart whose spectrum is harder. We stress that Fig. 4 has no direct phenomenological relevance, given that quark mass effects are poised to affect the shape of the Higgs transverse momentum distributions [81]. Even though we provide an estimate of these mass effects on the fully inclusive level, to obtain the correct high $p_{T}$-tail of the distribution, the quark mass effects in the EW amplitudes would also need to be considered. These amplitudes are beyond the current state of the art in multiloop computations.

We also report in Fig. 5 on the shape of the kinematic dependence of $\mathcal{M}_{g g \rightarrow H g}^{\left(\alpha_{s}^{3} \alpha^{2}\right)}$ relative to that of $\mathcal{M}_{g g \rightarrow H g}^{\left(\alpha_{s}^{3} \alpha\right)}$. We find that the dependence of this ratio on $z$ is more marked. In particular, we see that the ratio stabilizes rapidly as we approach the production threshold $z \rightarrow 1$, which amounts to the bulk of the relevant phase space. This is in line with the observation that factorization-like approximations provide a good approximation of the total cross section. For $l \sim 0.5$, the limit $z \rightarrow 0$ corresponds to larger values of the Higgs transverse momentum, where we see that the 


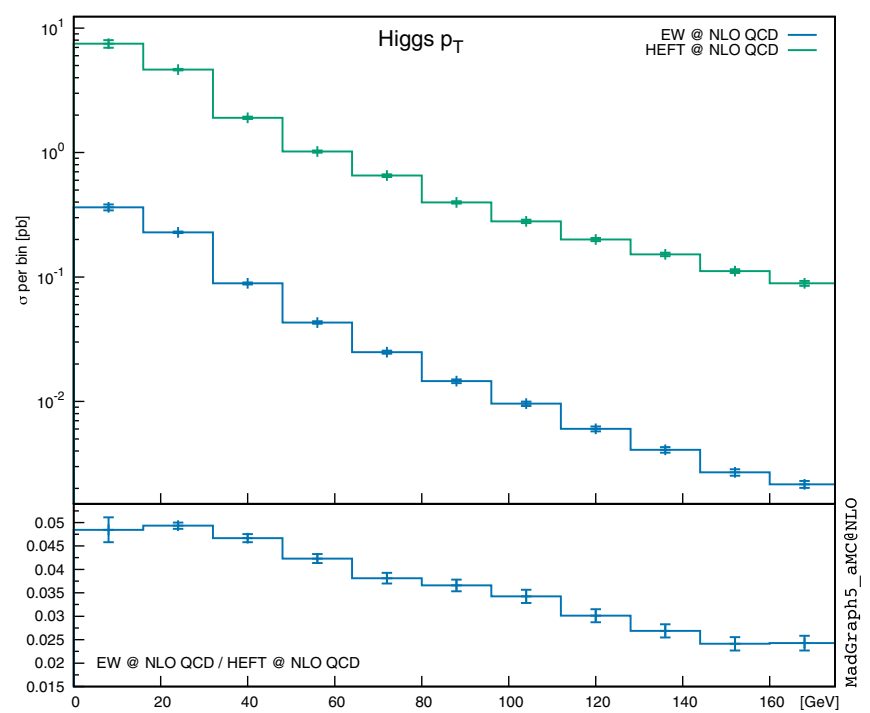

FIG. 4. Differential prediction for the $\mathcal{O}\left(\alpha_{s}^{3} \alpha^{2}\right)$ EW contribution to the Higgs transverse momentum distribution, compared to its LO HEFT counterpart.

two-loop EW matrix element is of smaller magnitude than that of its tree-level HEFT counterpart, as anticipated from the transverse momentum distribution shown in Fig. 4.

The factorization hypothesis of the Higgs cross section is

$$
\sigma_{g g \rightarrow H+X}^{\left(\sum_{i} \alpha_{s}^{i+2}\left(\alpha+\alpha^{2}\right)\right)}=C^{2}\left(\tilde{\sigma}_{g g \rightarrow H+X}^{\left(\alpha_{s}^{2} \alpha\right)}+\tilde{\sigma}_{g g \rightarrow H+X}^{\left(\alpha_{s}^{3} \alpha\right)}+\cdots\right)
$$

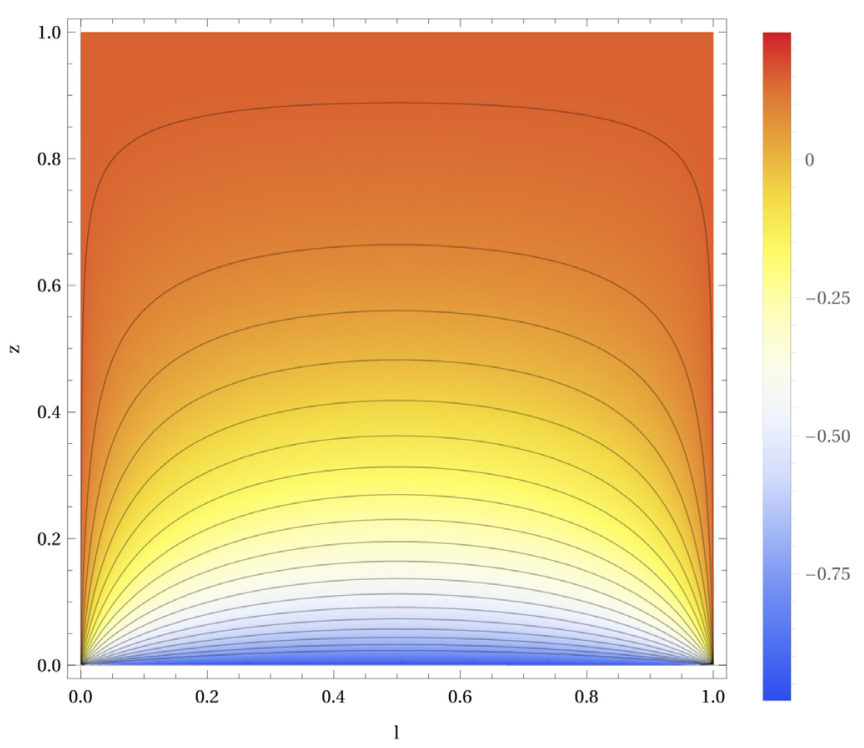

FIG. 5. Plot of the quantity $\frac{\left(\mathcal{M}_{g g \rightarrow H g}^{\left(\alpha_{s}^{3} \alpha^{2}\right)} / \mathcal{M}_{g g \rightarrow H g}^{\left(\alpha_{s}^{3} \alpha\right)}-R^{\mathrm{NLO}}\right)}{R^{\mathrm{NLO}}}$ with $R^{\mathrm{NLO}}=\sigma_{g g \rightarrow H+X}^{\left(\alpha_{s}^{3} \alpha^{2}\right)} / \sigma_{g g \rightarrow H+X}^{\left(\mathrm{HEFT}, \alpha_{s}^{3} \alpha\right)}$, in terms of the rescaled kinematic invariants $z=M_{H}^{2} / s$ and $l=t /\left(M_{H}^{2}-s\right)$, for a sample of $\sim 150000$ phase-space points. The lines of constant deviation span the range $[-0.75,0.15]$ in increments of 0.05 . with

$$
C=\sum_{i=0}\left(\frac{\alpha_{s}}{\pi}\right)^{i}\left(C_{\mathrm{QCD}}^{(i)}+\lambda_{\mathrm{EW}} C_{w}^{(i)}\right)
$$

and $C_{w}^{(0)}=C_{\mathrm{QCD}}^{(0)}=1, C_{\mathrm{QCD}}^{(1)}=11 / 4$. In terms of our quantities from Eqs. (1)-(4), we have

$$
\begin{gathered}
\tilde{\sigma}_{g g \rightarrow H+X}^{\left(\alpha_{s}^{2} \alpha\right)}=\sigma_{g g \rightarrow H+X}^{\left(\mathrm{HEFT}, \alpha_{s}^{2} \alpha\right)}, \\
\tilde{\sigma}_{g g \rightarrow H+X}^{\left(\alpha_{s}^{3} \alpha\right)}=-2 C_{\mathrm{QCD}}^{(1)} \sigma_{g g \rightarrow H+X}^{\left(\mathrm{HEFT}, \alpha_{s}^{2} \alpha\right)}+\sigma_{g g \rightarrow H+X}^{\left(\mathrm{HEFT}, \alpha_{s}^{3} \alpha\right)}, \\
\lambda_{\mathrm{EW}}=\frac{\sigma_{g g \rightarrow H+X}^{\left(\alpha_{s}^{2} \alpha^{2}\right)}}{2 \sigma_{g g \rightarrow H+X}^{\left(\mathrm{HEFT}, \alpha_{s}^{2} \alpha\right)}},
\end{gathered}
$$

$C_{w}^{(1)}=C_{\mathrm{QCD}}^{(1)}+\left(\frac{\alpha_{s}}{\pi}\right)^{-1}\left(\frac{\sigma_{g g \rightarrow H+X}^{\left(\alpha_{s}^{3} \alpha^{2}\right)}}{\sigma_{g g \rightarrow H+X}^{\left(\alpha_{s}^{2} \alpha^{2}\right)}}-\frac{\sigma_{g g \rightarrow H+X}^{\left(\mathrm{HEFT}, \alpha_{s}^{3} \alpha\right)}}{\sigma_{g g \rightarrow H+X}^{\left(\mathrm{HEFT}, \alpha_{s}^{2} \alpha\right)}}\right)$.

For $\mu_{R}=\mu_{F}$, our exact computation thus yields

$$
\lambda_{\mathrm{EW}}=0.026,
$$

$\left.C_{w}^{(1)}\right|_{\mu_{R}=\frac{1}{2} M_{H}}=-1.700,\left.\quad C_{w}^{(1)}\right|_{\mu_{R}=M_{H}}=-2.072$,

which is quite different from $C_{w}^{(1)}=7 / 6$ as estimated in the infinite boson-mass approximation; however, it is still within the uncertainty estimate of Ref. [24].

Remaining corrections to our computation: We identify three main contributions still unaccounted for in our computation of the mixed QCD-EW cross section. We provide here an estimate for each, together with an associated uncertainty:

(1) Heavy-quark (mass) effects in the QCD amplitudes can be estimated by using

$$
K_{(\mathrm{N}) \mathrm{LO}), M_{Q}}^{\mathrm{QCD}}=\frac{V_{\mathrm{FIN}, M_{Q}}^{((\mathrm{N}) \mathrm{LO})}}{V_{\mathrm{FIN}, \mathrm{HEFT}}^{((\mathrm{N}) \mathrm{LO})}}
$$

where the subscript $M_{Q}$ denotes the QCD background of a heavy quark of mass $M_{Q}, y_{Q}=M_{Q} / v$, and $V_{\text {FIN }}$ as defined in the Appendix. The twoloop virtual QCD amplitude is renormalized in a five-flavor decoupling scheme. We account for heavy quark-mass effects in the QCD amplitudes by rescaling with $K_{(\mathrm{N}) \mathrm{LO}), M_{Q}}^{\mathrm{QCD}}$, listed in Table II, and we assign an uncertainty of $\pm 50 \%$ of the estimated NLO effect based on the unknown (hard) realemission contributions.

(2) Top quark effects in the LO EW amplitudes were studied in Refs. $[44,45]$ and amount to $-1.8 \%$ of the LO cross section. We assign an uncertainty of the 
TABLE II. $\quad K$ factors defined as in Eq. (13) for different heavy quark masses $M_{Q}=M_{c}, M_{b}, M_{t}$.

\begin{tabular}{lcrr}
\hline \hline Quark & $M_{Q}[\mathrm{GeV}]$ & $K_{\mathrm{LO}, M_{Q}}^{\mathrm{QCD}}$ & $K_{\mathrm{NLO}, M_{Q}}^{\mathrm{QCD}}$ \\
\hline Charm & 1.3 & -0.010 & -0.018 \\
Bottom & 4.2 & -0.042 & -0.069 \\
Top & 173 & 1.032 & 1.031 \\
\hline \hline
\end{tabular}

same size based on the unknown top quark contribution in the NLO EW amplitudes.

(3) Higher-order QCD corrections can be accounted for under the assumption that the EW form factor receives the same QCD correction as the HEFT operator:

$$
\sigma_{g g \rightarrow H+X}^{\left(\alpha_{s}^{4}+\alpha_{s}^{5}\right) \alpha^{2}}=\sigma_{g g \rightarrow H+X}^{\left(\alpha_{s}^{2}+\alpha_{s}^{3}\right) \alpha^{2}} \frac{\sigma_{g g \rightarrow H+X}^{\left(\alpha_{s}^{4}+\alpha_{s}^{5}\right) \alpha}}{\sigma_{g g \rightarrow H+X}^{\left(\alpha_{s}^{2}+\alpha_{s}^{3}\right) \alpha}}
$$

and we assign an uncertainty $\Delta_{\text {fact }}$ as

$$
\Delta_{\mathrm{fact}}= \pm \delta_{\mathrm{fact}} \sigma_{g g \rightarrow H+X}^{\left(\alpha_{s}^{4}+\alpha_{s}^{5}\right) \alpha^{2}}
$$

with

$$
\begin{aligned}
& \delta_{\text {fact }}=\left|\frac{\frac{\sigma_{g g \rightarrow H+X}^{\left(\alpha_{s}^{2} \alpha^{2}\right)}}{\sigma_{g g \rightarrow H+X}^{\left(\mathrm{HEFT}, \alpha_{s}^{2} \alpha\right)}} \sigma_{g g \rightarrow H+X}^{\left(\mathrm{HEFT}, \alpha_{s}^{3} \alpha\right)}}{\sigma_{g g \rightarrow H+X}^{\left(\alpha_{s}^{3} \alpha^{2}\right)}}-1\right| \\
& =\left\{\begin{array}{ll}
0.15 ; & \mu_{R / F}=M_{H} \\
0.17 ; & \mu_{R / F}=\frac{M_{H}}{2}
\end{array},\right.
\end{aligned}
$$

based on testing this EW-QCD factorization hypothesis on our exact NLO correction. We compute the higher orders in QCD with the program iHixs 2 [82]. When combining the additional contributions and uncertainties above in the setup of Table I, we arrive at our best estimate for the EW contribution of gluon-initiated Higgs production:

$$
\begin{aligned}
& \sigma_{g g \rightarrow H+X}^{(\mathrm{EW}, \mathrm{best})}=\sigma_{g g \rightarrow H+X}^{\left(\mathrm{HEFT}, \alpha_{s}^{2} \alpha+\alpha_{s}^{3} \alpha\right)} \times(4.81 \% \quad \text { (our computation) } \\
&+0.15 \pm 0.04 \% \text { (top mass effects in QCD amp) } \\
&-0.27 \pm 0.09 \% \quad \text { (bottom quark effects in QCD amp) } \\
&-0.07 \pm 0.02 \% \text { (charm quark effects in QCD amp) } \\
&-0.04 \pm 0.04 \% \text { (top quark effects in EW) } \\
&+2.5 \pm 0.4 \%(\mathrm{QCD} \text { higher orders) }) \\
&= \sigma_{g g \rightarrow H+X}^{\left(\mathrm{HEFT}, \alpha_{s}^{2} \alpha+\alpha_{s}^{3} \alpha\right)} \times(7.11 \pm 0.6 \%) \\
&= 2.17 \pm 0.18 \mathrm{pb} .
\end{aligned}
$$

A similar computation for $\mu_{R / F}=\frac{1}{2} M_{H}$ yields $\sigma_{g g \rightarrow H+X}^{\text {(EW,best) }}=$ $2.02 \pm 0.14 \mathrm{pb}$.

Quark-induced and other EW contributions: The PDF suppression from $q q$ production channels renders them negligible, and we only consider here $q g$-induced channels. We identify the following two categories of quark-induced contributions:

(1) One-loop EW contributions start at $\mathcal{O}\left(\alpha_{s}^{2} \alpha^{2}\right)$ for the process $q g \rightarrow H q$ and involve one-loop EW triangle and box diagrams interfering with the one-loop QCD Higgs amplitude with exact top and bottom quark mass dependence. They can be computed exactly using the loop-induced module [83] of MG5aMC together with the loop-ready EW UFO model of Ref. [84]. We, however, use here the result of Table 3 of Ref. [47], which includes all one-loop EW contributions in the $g g$ and $q g$ channels (including Higgs-strahlung), and we refer to them for drawings of the relevant diagram classes. We assign a theoretical uncertainty to this contribution based on the $38 \% \mu_{R}$ scale variation of its $q g$ component of order $\mathcal{O}\left(\alpha_{s}^{2} \alpha^{2}\right)$, and we obtain

$$
\sigma_{1 \text { loop }}^{(\mathrm{EW})}=\left\{\begin{array}{ll}
0.025 \pm 0.052 \mathrm{pb} ; & \mu_{R / F}=M_{H} \\
0.031 \pm 0.062 \mathrm{pb} ; & \mu_{R / F}=\frac{M_{H}}{2}
\end{array} .\right.
$$

The large uncertainty assigned here reflects the accidental cancellation found among all contributions considered in Ref. [47].

(2) Quark corrections to EW form factors correspond to $q g$ contributions stemming from the QCD evolution of the initial-state gluon entering the EW two-loop form factor as shown in Fig. 6. We estimate this contribution by rescaling our LO mixed QCD-EW result by the $q g K$ factor of the HEFT contribution. This rescaling does not capture the nonuniversal unknown (hard) real emission contribution, and we assign 30\% uncertainty based on these effects at NLO. However, the factorization-scale dependence of the $q g$ channel will be captured well, since it effectively amounts to replacing the reduced matrix element, as indicated in Fig. 6. After combining both $q g$ and $g g$ channels, the factorization-scale dependence is reduced, and we assign an uncertainty of $10 \%$ based on the relative difference of the $\mu_{F}$ dependence of the NLO EW and NLO HEFT gluoninduced contributions. To estimate the higher-order QCD corrections, we perform the same rescaling and assign an uncertainty of $50 \%$ based on the fact that we do not know if they are captured well by a factorization ansatz. We obtain

$$
\sigma_{q g \rightarrow H+X}^{(\mathrm{EW})}=\left\{\begin{array}{ll}
-0.10 \pm 0.05 \mathrm{pb} ; & \mu_{R / F}=M_{H} \\
0.12 \pm 0.05 \mathrm{pb} ; & \mu_{R / F}=\frac{M_{H}}{2}
\end{array},\right.
$$




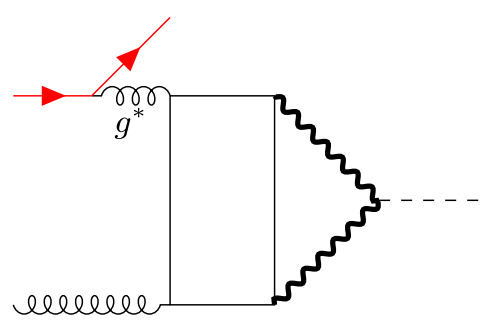

FIG. 6. Representative diagram of the $q g$-initiated contribution to the NLO mixed EW-QCD cross section. Curly lines denote gluons, wavy lines correspond to massive weak gauge bosons, continuous straight lines denote massless quarks, and the dashed line represents the Higgs boson.

where we used the program iHixs 2 to compute the QCD corrections beyond NLO. Notice that, as expected, including $\sigma_{q g \rightarrow H+X}^{(\mathrm{EW})}$ helps to reduce the difference in the quantity $\sigma_{g g \rightarrow H+X}^{(\mathrm{EW}, \text { best })}$ when computed with $\mu_{F}=M_{H}$ and $\mu_{F}=\frac{M_{H}}{2}$.

(3) Higher-order EW contributions are estimated by reweighting our cross section with

$$
K_{(\mathrm{N}) \mathrm{LO})}^{\text {pure-EW }}=\frac{V_{\text {FIN,pure-EW }}^{((\mathrm{N}) \mathrm{LO})}}{V_{\text {FIN,mixed-QCD-EW }}^{((\mathrm{N}) \mathrm{LO})}},
$$

where we compute $V_{\text {FIN }, E W}^{((\mathrm{N}) \mathrm{LO})}$ by squaring (interfering) the relevant two- and three-loop EW virtual amplitudes and assign a 50\% uncertainty on the NLO result based on the unknown (hard) real radiation:

$$
\sigma_{g g \rightarrow H+X}^{(\text {squared EW })}=\left\{\begin{array}{ll}
0.018 \pm 0.005 \mathrm{pb} ; & \mu_{R / F}=M_{H} \\
0.020 \pm 0.005 \mathrm{pb} ; & \mu_{R / F}=\frac{M_{H}}{2}
\end{array} .\right.
$$

Together with the gluon-induced contributions of Eq. (18), we finally construct our best estimate for the overall contributions of EW origin to Higgs hadroproduction with the parameters given in Table I:

$$
\begin{aligned}
& \sigma_{p p \rightarrow H+X}^{(\mathrm{EW}, \text { best })} \\
& =\left\{\begin{array}{l}
(6.91 \pm 0.9 \%) \times \sigma_{g g \rightarrow H+X}^{\left(\mathrm{HEFT}, \alpha_{s}^{2} \alpha+\alpha_{s}^{3} \alpha\right)} ; \quad \mu_{R / F}=M_{H} \\
(6.43 \pm 0.8 \%) \times \sigma_{g g \rightarrow H+X}^{\left(\mathrm{HEFT}, \alpha_{s}^{2} \alpha+\alpha_{s}^{3} \alpha\right)} ; \quad \mu_{R / F}=\frac{M_{H}}{2}
\end{array}\right. \\
& =\left\{\begin{array}{l}
2.11 \pm 0.28 \text { (theory) pb; } \mu_{R / F}=M_{H} \\
2.19 \pm 0.26 \text { (theory) pb; } \mu_{R / F}=\frac{M_{H}}{2}
\end{array}\right.
\end{aligned}
$$

\section{CONCLUSION}

We evaluated the NLO QCD correction to the mixed EW-QCD light-quark contribution to Higgs production via gluon fusion. Unlike previous computations of this quantity, we retained the exact dependence on the weak boson masses. The two-loop real-emission amplitudes were computed by solving differential equations for the relevant scalar integrals at runtime in terms of onedimensional generalized power series. We implemented the resulting matrix elements in a flexible manner by encoding them as form factors of a UFO model, which we made publicly available as a MadGraph5_aMC@NLO plugin. We performed the phase-space integration numerically in two separate implementations of the FKS and COLORFUL subtraction scheme using an offline parallelization model in order to accommodate the evaluation speed of our matrix elements.

We presented the distribution of the Higgs rapidity, which shows a flat differential $K$-factor, and of the Higgs transverse momentum, whose spectrum is softer than its HEFT counterpart when ignoring quark mass effects.

When related to the NLO-accurate HEFT cross section, we find the EW contribution from light quarks to be 5.1\% for $\mu_{R}=\mu_{F}=\frac{1}{2} M_{H}$, which is very close to the result of $5.2 \%$ obtained in the infinite weak boson mass limit $[20,24,50]$. Our result therefore allows us to reduce the uncertainty of $\pm 1 \%$ assigned thus far to the mixed QCDEW contribution in order to reflect the absence of an exact weak boson mass treatment at NLO. We also conclude that violations to the factorization of $\mathrm{EW}$ and $\mathrm{QCD}$ contributions are modest. This gives further confidence in the rescaling of our cross section with higher-order QCD corrections computed in HEFT which, together with the estimate of other partially unknown effects, yields our best estimate of $6.91 \pm 0.93 \%$ (relative to the gluon-induced NLO HEFT) for the overall contribution of EW origin to Higgs production at the LHC. The LO and NLO quarkinduced EW contributions now stand as an important remaining source of uncertainty, and they can be computed in the future using the same methods as those presented in this work.

\section{ACKNOWLEDGMENTS}

This project has received funding from the European Research Council (ERC) under Grant Agreement No. 694712 (PertQCD) and the Swiss National Science Foundation (SNF) under Contract Agreement No. 177632. We thank S. Abreu for useful discussions about cut Feynman integrals, N. Deutschmann for his collaboration on the development of the implementation of the COLORFUL subtraction and many useful discussions, A. Ochirov for insightful discussions about helicity amplitudes, A. Lazopoulos for useful discussion regarding iHixs, and C. Anastasiou for his valuable feedback on the draft.

\section{APPENDIX: BENCHMARK NUMERICAL MATRIX ELEMENT EVALUATIONS}

In order to facilitate the reproduction of our work, we report here benchmark numerical results of our matrix 
elements, summed/averaged over helicity and color configurations, evaluated for specific kinematic configurations with $\alpha_{S}\left(M_{Z}\right)=0.118$ and all other SM parameters as in Table I. For the virtual matrix element, we only report the finite part as defined in Eq. (B.2) of Ref. [73] or Eq. (A.1) of Ref. [85]. The $g g \rightarrow H$ matrix elements are

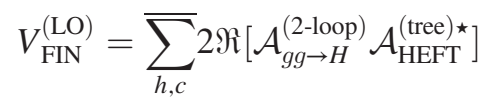

$$
\begin{aligned}
& =5.1508192663885 \times 10^{-4}\left[\mathrm{GeV}^{2}\right] \text {, }
\end{aligned}
$$

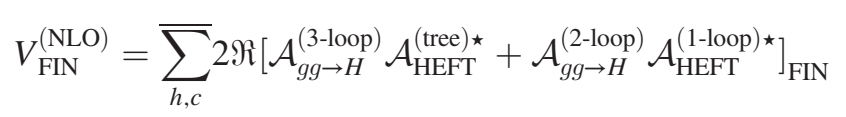

$$
\begin{aligned}
& =3.6824078313996 \times 10^{-4}\left[\mathrm{GeV}^{2}\right] \text {. }
\end{aligned}
$$

We also provide the relation of $V_{\mathrm{FIN}}^{(\mathrm{NLO})}$ above with the matrix element $M_{\mathrm{FIN}, I_{1}}$ obtained from the finite amplitudes computed with the methods of Refs. [48,72]:

$$
V_{\mathrm{FIN}}^{(\mathrm{NLO})}=M_{\mathrm{FIN}, I_{1}}+C_{A} \pi^{2} V_{\mathrm{FIN}}^{(\mathrm{LO})},
$$

where $C_{A}=3$ denotes the number of colors. Finally, we give the two-loop $\mathrm{gg} \rightarrow \mathrm{Hg}$ matrix elements evaluated at two different benchmark kinematic points specified with the two Mandelstam invariants $s=\left(p_{g_{1}}+p_{g_{2}}\right)^{2}$ and $t=\left(p_{g_{2}}-p_{H}\right)^{2}$ :

$$
\begin{aligned}
& \sum_{h, c} 2 \Re\left[\mathcal{A}_{g g \rightarrow H g}^{(2-\text { loop })} \mathcal{A}_{\mathrm{HEFT}}^{(\mathrm{tree}) \star}\right]\left(s=5 M_{H}^{2}, t=-3 M_{H}^{2}\right) \\
& =9.0303320385123 \times 10^{-6}\left[\mathrm{GeV}^{0}\right], \\
& \sum_{h, c} 2 \Re\left[\mathcal{A}_{g g \rightarrow H g}^{(2-\text { loop })} \mathcal{A}_{\mathrm{HEFT}}^{(\text {tree })}\right]\left(s=5 M_{H}^{2}, t=-2 M_{H}^{2}\right)
\end{aligned}
$$$$
=5.8988801633472 \times 10^{-6}\left[\mathrm{GeV}^{0}\right] .
$$

[1] G. Aad et al. (ATLAS Collaboration), Phys. Lett. B 716, 1 (2012).

[2] S. Chatrchyan et al. (CMS Collaboration), Phys. Lett. B 716, 30 (2012).

[3] R. V. Harlander and T. Neumann, Phys. Rev. D 88, 074015 (2013).

[4] A. Banfi, A. Martin, and V. Sanz, J. High Energy Phys. 08 (2014) 053.

[5] A. Azatov and A. Paul, J. High Energy Phys. 01 (2014) 014.

[6] C. Grojean, E. Salvioni, M. Schlaffer, and A. Weiler, J. High Energy Phys. 05 (2014) 022.

[7] M. Schlaffer, M. Spannowsky, M. Takeuchi, A. Weiler, and C. Wymant, Eur. Phys. J. C 74, 3120 (2014).

[8] M. Buschmann, C. Englert, D. Goncalves, T. Plehn, and M. Spannowsky, Phys. Rev. D 90, 013010 (2014).

[9] S. Dawson, I. M. Lewis, and M. Zeng, Phys. Rev. D 90, 093007 (2014).

[10] M. Buschmann, D. Goncalves, S. Kuttimalai, M. Schonherr, F. Krauss, and T. Plehn, J. High Energy Phys. 02 (2015) 038.

[11] D. Ghosh and M. Wiebusch, Phys. Rev. D 91, 031701 (2015).

[12] S. Dawson, I. M. Lewis, and M. Zeng, Phys. Rev. D 91, 074012 (2015).

[13] U. Langenegger, M. Spira, and I. Strebel, arXiv:1507 .01373 .

[14] A. Azatov, C. Grojean, A. Paul, and E. Salvioni, J. High Energy Phys. 09 (2016) 123.

[15] M. Grazzini, A. Ilnicka, M. Spira, and M. Wiesemann, J. High Energy Phys. 03 (2017) 115.

[16] H. Georgi, S. Glashow, M. Machacek, and D. V. Nanopoulos, Phys. Rev. Lett. 40, 692 (1978).
[17] D. Graudenz, M. Spira, and P. M. Zerwas, Phys. Rev. Lett. 70, 1372 (1993).

[18] M. Spira, A. Djouadi, D. Graudenz, and P. M. Zerwas, Nucl. Phys. B453, 17 (1995).

[19] R. V. Harlander and W. B. Kilgore, Phys. Rev. Lett. 88, 201801 (2002).

[20] C. Anastasiou and K. Melnikov, Nucl. Phys. B646, 220 (2002).

[21] V. Ravindran, J. Smith, and W. L. van Neerven, Nucl. Phys. B665, 325 (2003).

[22] C. Anastasiou, C. Duhr, F. Dulat, F. Herzog, and B. Mistlberger, Phys. Rev. Lett. 114, 212001 (2015).

[23] B. Mistlberger, J. High Energy Phys. 05 (2018) 028.

[24] C. Anastasiou, C. Duhr, F. Dulat, E. Furlan, T. Gehrmann, F. Herzog, A. Lazopoulos, and B. Mistlberger, J. High Energy Phys. 05 (2016) 058.

[25] R. Harlander and P. Kant, J. High Energy Phys. 12 (2005) 015.

[26] C. Anastasiou, S. Beerli, S. Bucherer, A. Daleo, and Z. Kunszt, J. High Energy Phys. 01 (2007) 082.

[27] U. Aglietti, R. Bonciani, G. Degrassi, and A. Vicini, J. High Energy Phys. 01 (2007) 021.

[28] R. Bonciani, G. Degrassi, and A. Vicini, J. High Energy Phys. 11 (2007) 095.

[29] C. Anastasiou, S. Bucherer, and Z. Kunszt, J. High Energy Phys. 10 (2009) 068.

[30] C. Anastasiou, N. Deutschmann, and A. Schweitzer, J. High Energy Phys. 07 (2020) 113.

[31] R. V. Harlander, M. Prausa, and J. Usovitsch, J. High Energy Phys. 10 (2019) 148.

[32] M. L. Czakon and M. Niggetiedt, J. High Energy Phys. 05 (2020) 149. 
[33] M. Prausa and J. Usovitsch, arXiv:2008.11641.

[34] R. Bonciani, V. Del Duca, H. Frellesvig, J. M. Henn, F. Moriello, and V.A. Smirnov, J. High Energy Phys. 12 (2016) 096.

[35] R. Bonciani, V. Del Duca, H. Frellesvig, J. Henn, M. Hidding, L. Maestri, F. Moriello, G. Salvatori, and V. Smirnov, J. High Energy Phys. 01 (2020) 132.

[36] H. Frellesvig, M. Hidding, L. Maestri, F. Moriello, and G. Salvatori, J. High Energy Phys. 06 (2020) 093.

[37] V. Del Duca, W. Kilgore, C. Oleari, C. Schmidt, and D. Zeppenfeld, Nucl. Phys. B616, 367 (2001).

[38] L. Budge, J. M. Campbell, G. De Laurentis, R. K. Ellis, and S. Seth, J. High Energy Phys. 05 (2020) 079.

[39] R. V. Harlander and K. J. Ozeren, J. High Energy Phys. 11 (2009) 088.

[40] A. Pak, M. Rogal, and M. Steinhauser, J. High Energy Phys. 02 (2010) 025.

[41] R. V. Harlander, T. Neumann, K. J. Ozeren, and M. Wiesemann, J. High Energy Phys. 08 (2012) 139.

[42] U. Aglietti, R. Bonciani, G. Degrassi, and A. Vicini, Phys. Lett. B 595, 432 (2004).

[43] U. Aglietti, R. Bonciani, G. Degrassi, and A. Vicini, Phys. Lett. B 600, 57 (2004).

[44] G. Degrassi and F. Maltoni, Phys. Lett. B 600, 255 (2004).

[45] S. Actis, G. Passarino, C. Sturm, and S. Uccirati, Phys. Lett. B 670, 12 (2008).

[46] C. Anastasiou, R. Boughezal, and F. Petriello, J. High Energy Phys. 04 (2009) 003.

[47] V. Hirschi, S. Lionetti, and A. Schweitzer, J. High Energy Phys. 05 (2019) 002.

[48] M. Bonetti, K. Melnikov, and L. Tancredi, Nucl. Phys. B916, 709 (2017).

[49] M. Bonetti, K. Melnikov, and L. Tancredi, Phys. Rev. D 97 , 056017 (2018); 97, 099906(E) (2018).

[50] C. Anastasiou, V. del Duca, E. Furlan, B. Mistlberger, F. Moriello, A. Schweitzer, and C. Specchia, J. High Energy Phys. 03 (2019) 162.

[51] M. Becchetti, R. Bonciani, V. Casconi, V. Del Duca, and F. Moriello, J. High Energy Phys. 12 (2018) 019.

[52] M. Bonetti, E. Panzer, V. A. Smirnov, and L. Tancredi, J. High Energy Phys. 11 (2020) 045.

[53] F. Moriello, J. High Energy Phys. 01 (2020) 150.

[54] P. Maierhoefer, J. Usovitsch, and P. Uwer, Comput. Phys. Commun. 230, 99 (2018).

[55] A. V. Smirnov, Comput. Phys. Commun. 189, 182 (2015).

[56] R. N. Lee, J. Phys. Conf. Ser. 523, 012059 (2014).

[57] K. Chetyrkin and F. Tkachov, Nucl. Phys. B192, 159 (1981).

[58] F. Tkachov, Phys. Lett. 100B, 65 (1981).
[59] S. Laporta, Int. J. Mod. Phys. A 15, 5087 (2000).

[60] T. Gehrmann and E. Remiddi, Nucl. Phys. B580, 485 (2000).

[61] A. Kotikov, Phys. Lett. B 254, 158 (1991).

[62] E. Remiddi, Nuovo Cimento A 110, 1435 (1997).

[63] T. Gehrmann and E. Remiddi, Nucl. Phys. B640, 379 (2002).

[64] M. Argeri and P. Mastrolia, Int. J. Mod. Phys. A 22, 4375 (2007).

[65] J. M. Henn, Phys. Rev. Lett. 110, 251601 (2013).

[66] J. M. Henn, J. Phys. A 48, 153001 (2015).

[67] M. Argeri, S. Di Vita, P. Mastrolia, E. Mirabella, J. Schlenk, U. Schubert, and L. Tancredi, J. High Energy Phys. 03 (2014) 082.

[68] R. N. Lee, J. High Energy Phys. 04 (2015) 108.

[69] A. Georgoudis, K. J. Larsen, and Y. Zhang, Comput. Phys. Commun. 221, 203 (2017).

[70] T. Gehrmann, A. von Manteuffel, L. Tancredi, and E. Weihs, J. High Energy Phys. 06 (2014) 032.

[71] M. Becchetti and R. Bonciani, J. High Energy Phys. 01 (2018) 048.

[72] M. Bonetti, K. Melnikov, and L. Tancredi, Phys. Rev. D 97, 034004 (2018).

[73] R. Frederix, S. Frixione, F. Maltoni, and T. Stelzer, J. High Energy Phys. 10 (2009) 003.

[74] S. Lionetti, Ph.D. thesis, ETH, 2018.

[75] V. Del Duca, N. Deutschmann, and S. Lionetti, J. High Energy Phys. 12 (2019) 129.

[76] G. Somogyi and Z. Trocsanyi, arXiv:hep-ph/0609041.

[77] G. Somogyi, J. High Energy Phys. 05 (2009) 016.

[78] C. Degrande, C. Duhr, B. Fuks, D. Grellscheid, O. Mattelaer, and T. Reiter, Comput. Phys. Commun. 183, 1201 (2012).

[79] J. Alwall, R. Frederix, S. Frixione, V. Hirschi, F. Maltoni, O. Mattelaer, H. S. Shao, T. Stelzer, P. Torrielli, and M. Zaro, J. High Energy Phys. 07 (2014) 079.

[80] M. Whalley, D. Bourilkov, and R. Group, in HERA and the LHC: A Workshop on the Implications of HERA and LHC Physics (Startup Meeting, CERN, 2004; Midterm Meeting, CERN, 2004) (2005), pp. 575-581.

[81] K. Becker et al., arXiv:2005.07762.

[82] F. Dulat, A. Lazopoulos, and B. Mistlberger, Comput. Phys. Commun. 233, 243 (2018).

[83] V. Hirschi and O. Mattelaer, J. High Energy Phys. 10 (2015) 146.

[84] R. Frederix, S. Frixione, V. Hirschi, D. Pagani, H.-S. Shao, and M. Zaro, J. High Energy Phys. 07 (2018) 185.

[85] V. Hirschi, R. Frederix, S. Frixione, M. V. Garzelli, F. Maltoni, and R. Pittau, J. High Energy Phys. 05 (2011) 044. 